\title{
Estudio preliminar comparativo entre los acondicionadores de suelos convencionales y las escorias del proceso Conox para uso agrícola $^{(\cdot)}$
}

\author{
O. Tapia ${ }^{(*)}$, L. Flores ${ }^{(* *)}$, G. Barrera ${ }^{(* * *)}$ y A. Formoso ${ }^{(* * * *)}$ \\ Resumen El presente estudio tiene como finalidad explorar el comportamiento físico-químico y estructural de \\ sustancias convencionales de base calcio, empleadas comúnmente como estabilizadores de suelos, en \\ comparación con el uso de residuos de origen siderúrgico (escorias de aceración del proceso \\ Conox), para el tratamiento de suelos de cultivo de la zona Norte del Estado de Michoacán. Este tra- \\ bajo evidenció que las escorias promueven el carácter químico de los suelos, pero en un grado menor \\ que los aditivos comerciales. No obstante, las primeras proporcionan una mayor aportación en ele- \\ mentos micronutrientes necesarios para el crecimiento del vegetal.
}

Palabras clave: Suelos agrícolas. Escorias. Proceso Conox.

\section{Comparative and preliminary study of the conventional soil conditioners and the Conox process slags for the agricultural use}

\begin{abstract}
The object of this preliminary study is the measurement of the physical-chemistry and structural properties of commercial conditioners used in agricultural soils at the North of Michoacán, México. These materials were compared to the Conox slags used for the same purpose. The results showed that the first materials changed the chemical character of the soil, however the slags in the soil increase the digest elements concentration more than commercial conditioners.
\end{abstract}

Keywords: Agricultural soils. Slags. Conox process.

\section{INTRODUCCIÓN}

En la actualidad, las crecientes restricciones en cuanto a la protección del medio ambiente a nivel industrial ocasionan cambios en las empresas en el sentido de hacer más eficiente los procesos productivos con el máximo aprovechamiento de sus residuos. Lo anterior conduce a un verdadero fortalecimiento del ámbito ecológico industrial (1-3).

\footnotetext{
$(\bullet \quad$ Trabajo recibido el día 22 de enero de 1998 y aceptado en su forma final el 23 de octubre de 1998.

(*) Instituto Tecnológico de Morelia. Av. Tecnológico 1500. Colonia Lomas de Santiaguito. Morelia. Mich. 58120 (México).

(**) Tubos de Acero de México, S.A.

(***) Instituto Tecnológico Agropecuario núm. 7.

(****) Centro Nacional de Investigaciones Metalúrgicas, CENIM (CSIC). Av. Gregorio del Amo, 8. 28040Madrid (España)
}

Uno de los aspectos importantes hoy en día, de toda empresa integrada, y que incide significativamente en el sector siderúrgico mexicano, es la reutilización de sus subproductos (escorias), ya sea retornándolos al propio proceso, o bien de manera alternativa como un coproducto hacia otros sectores, como es el caso que se trata.

En particular, la empresa SICARTSA genera anualmente un volumen de $185.400 \mathrm{t}$ de escoria procedentes del proceso Conox, que puede utilizarse en la modificación del carácter químico $(\mathrm{pH})$ de los suelos de cultivo. Conforme a lo antes expresado, se ha manifestado un marcado interés por parte de los países del continente europeo e incluso de los Estados Unidos de Norteamérica, en organizarse de manera coordinada para proporcionarle a este subproducto un determinado valor agregado (4-6).

Por otra parte, diversos investigadores (7-29 y 34-44), han dirigido sus estudios fundamentales 
hacia el conocimiento, tanto de las soluciones moleculares de escoria como de las electrodisociadas, en donde no sólo se explicita el comportamiento de las especies iónicas en cuanto a sus características dimensionales, potencial de ionización, electronegatividad, etc, sino también se abordan aspectos tales como basicidad, cristaloquímica de los constituyentes en la escoria y depolimerización de estos componentes.

Por lo que respecta a los suelos de cultivo, informes de la bibliografía (30-33 y 45-49) manifiestan que en virtud de su estado mineralógico, características fisicoquímicas y edafológicas, pueden ser tratados con residuos del proceso de escorificación de oxiconvertidores.

\section{PARTE EXPERIMENTAL}

La experimentación a nivel laboratorio se fundamenta en la secuencia metodológica siguiente: Inicialmente, la escoria se concentró mediante separación magnética para eliminar los restos de acero y se clasificó granulométricamente para obtener una fracción adecuada para su uso agrícola. A continuación, se procedió a determinar la composición y fases mineralógicas de muestras de escoria, procedentes directamente del horno y de los patios de almacenamiento, mediante espectrometría y difractometría de rayos X. Para ello, se molieron $50 \mathrm{~g}$ de muestra en un molino de disco Mca. Herzog, Mod. HSM250, para después situarse en el portamuestras del espectrómetro de rayos X simultáneo multicanal Mca. Siemens, Mod. MRS-404. De la forma antes descrita se utilizó también un difractómetro Mca. Philips, el cual trabajó en las condiciones de $2 \% / \mathrm{min}$, $35 \mathrm{kV}$ y $15 \mathrm{~mA}$. Cabe mencionar que en estos resultados no se evaluaron los contenidos de cal libre sino sólo la concentración de $\mathrm{Ca} 0$ en la solución de escoria.

Por otra parte, la composición referencial de la escoria se obtuvo de la bibliografía (26). Para la determinación de las propiedades físicas se utilizó el molino antes indicado, en donde se obtuvo un producto fino que posteriormente se concentró y clasificó granulométricamente a -270 mallas (menor de $53 \mu \mathrm{m}$ ) a fin de ofrecer una mayor superficie de contacto. Se empleó un picnómetro Mca. Beckman, Mod. 930 y un equipo Fisher, en la determinación de la densidad y superficie específica, tanto para los materiales alcalinizantes (cal agrícola, hidróxido y carbonato de calcio), como para el propio suelo y residuo siderúrgico ensayado. Posteriormente, se valoró la variación del $\mathrm{pH}$ que ofrecen estos materiales en un medio acuoso. Para ello, se seleccionaron dos muestras, una de escoria y otra de material acondicionante (cal agrícola), con un peso de $100 \mathrm{~g}$ cada una. Estas muestras se pusieron en recipentes y se mezclaron con $500 \mathrm{ml}$ de agua. Para esta medición se utilizó un potenciómetro digital manual Mca. Courning, Mod. PS-15 durante un período que osciló de $3 \mathrm{~h}$ a 26 días, empleando dos soluciones buffer ( $\mathrm{pH}$ de 7 y 10) como medio de calibración. Posteriormente, se determinó el análisis químico de la solución de escoria y la del líquido sobrenadante en ella. Enseguida se evaluó el comportamiento del $\mathrm{pH}$ del suelo en estudio, con las adiciones antes mencionadas, definiéndose la proporción de aportadores de micronutrientes a éste, por parte de los aditivos utilizados. Para este experimento se calculó la cantidad equivalente de material acondicionante para 1, 2, 4 y 8 t/ha, bajo el criterio de considerar $50 \mathrm{~g}$ de suelo cultivable tomado a una profundidad de $20 \mathrm{~cm}$ y con una densidad de $10^{3} \mathrm{k} / \mathrm{m}^{3}$. Las muestras de suelo se molieron y se las agregó $100 \mathrm{ml}$ de agua destilada, así como los diferentes materiales ensayados. Posteriormente, se agitaron en una mesa de homogeneización y se midió el $\mathrm{pH}$ de la solución agitando periódicamente durante 1-24 días, hasta que el comportamiento de las curvas resultantes se estabilizara. Después, se analizó el potasio del suelo de cultivo con un analizador digital de llama Mca. Cole-Parmer, Mod. 2655-09. En cambio, para los elementos fósforo, hierro y manganeso se empleó un colorímetro Mca. Milton Roy, Mod. Spectronic 21D. Finalmente, el calcio y el magnesio se determinaron por el método de complejometría (titulación) con la técnica EDTA (acido etilen diamin tetra acetato).

\section{RESULTADOS Y DISCUSIÓN}

En el momento del desarrollo del estudio, la fuente informativa del Instituto Nacional de Estadística, Geografía e Informática (INEGI-58-59) no contaba con el mosaico edafológico integral del Estado de Michoacán. Por tal motivo, se consultaron 18 cartas de este tema para su conjunción. Posteriormente, se obtuvieron muestras de suelo virgen, de la zona denominada Ciénega de Chapala, correspondiente al Municipio de Santiago Tangamandapio, Mich., para su caracterización posterior.

El mosaico obtenido en cuanto a las características edafológicas del Estado demostraron la existencia de diferentes grados de acidez en los suelos de cultivo, a saber: Moderado ácido $(3,9-5,9)$ a poco ácido entre el rango de (5,91-6,99). Conforme a lo anterior, las zonas de mayor extensión están constituidas por el carácter químico antes citado, y por ello el interés del presente estudio.

Ahora bien, con relación a las escorias del proceso Conox, éstas se sujetaron a un acondicionamiento magnético-mecánico con la finalidad de eliminar las partículas ferrosas que provocan incremento en la capacidad de disolución del sistema 
escoria-suelo. Asimismo, estos residuos se tipificaron por espectrometría y difractometría de rayos $\mathrm{X}$. En la tabla I, se compara la composición de estas escorias con relación a una de tipo referencial.

De esta información se destaca que los contenidos de $\mathrm{SiO}_{2}$ y $\mathrm{Al}_{2} \mathrm{O}_{3}$ son inferiores a los reportados en la composición referencial, no siendo así para el caso del $\mathrm{CaO}$ y $\mathrm{FeO}$, por las propias condiciones de cal en exceso que permiten una mayor basicidad y capacidad de oxidación durante la escorificación. El contenido de fósforo se mantiene por debajo de lo señalado, en virtud de que las escorias europeas presentan más altos contenidos en este elemento por motivo de sus materias primas. En cuanto a las concentraciones de $\mathrm{MnO}$ y $\mathrm{MgO}$, estos se encuentran cercanos a la composición referencial.

$\mathrm{El}$ análisis mediante difracción de rayos $\mathrm{X}$ indica la presencia de monticellita (CMS) y wustita (F), productos de reacción de la etapa oxidante. Estas estructuras se encuentran asociadas a la ferrita de calcio $\left(\mathrm{C}_{2} \mathrm{~F}_{2}\right)$ y a los silicatos dobles de este elemento $\left(\mathrm{C}_{2} \mathrm{~S}\right)$, lo cual implica un cambio polimórfico de $\beta$ - $\gamma$ como resultado del efecto limitante de la asimilación de la cal en la solución de escoria y enfriamiento de ésta, que provoca un cambio volumétrico por razones hidratantes. El resto de las estructuras presentes en los materiales alcalinizantes destacaron la existencia de calcita y residuos de sílice; en cambio, en la cal hidratada se manifestó la estructura portlandita e igualmente la calcita, según se muestra en la figura 1.

Con relación al estudio microestructural, se observó la aparición de una matriz wustítica con oquedades, producto del enfriamiento lento que experimentó la escoria al ser extraída directamente del reactor. En cambio, las muestras obtenidas de los patios de almacenamiento manifestaron estructuras del tipo $\mathrm{C}_{2} \mathrm{~S}$, en una matriz wustítica. Conforme a lo anterior, no se revela el resto de las estructuras, debido al enmascaramiento existente en el campo focal.

De igual manera se determinaron las propiedades físicas (densidad y superficie específica) del

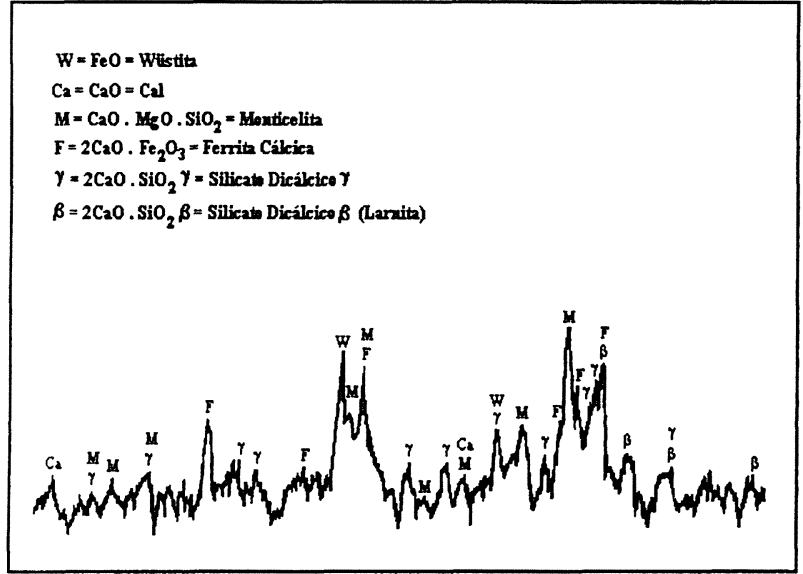

FIG. 1.- Fases estructurales de escorias Conox determinadas por difracción de rayos X.

Fig. 1.- Structural phases of Conox slags determined by $X$-ray diffraction

sistema en estudio. Los resultados se indican en la tabla II.

De estos resultados es evidente que el parámetro densidad, tanto en suelo como en los materiales alcalinizantes, se mantiene en promedio de 2,2 $\mathrm{g} / \mathrm{cm}^{3}$, no siendo así para la escoria, en donde dicho valor se incrementa a niveles de $3 \mathrm{~g} / \mathrm{cm}^{3}$, lo cual provocaría un descenso en la capacidad de disolución en el sistema.

Por otro lado, el efecto de la superficie específica se mostró significativamente notable para el caso de la cal hidratada, no siendo de igual forma para el resto de los materiales, donde se comportó oscilante $\left(0,8-4,5 \mathrm{~m}^{2} / \mathrm{g}\right)$. La escoria en particular presentó una superficie muy pequeña, quedando de manifiesto la necesidad de incrementar el tamaño granulométrico (-270 mallas), como se indica en la figura 2.

Con relación a la determinación del $\mathrm{pH}$ de soluciones acuosas de escoria y de cal agrícola, se observó que esta última eleva el pH de 7-12 a partir del lapso comprendido entre 2 y 26 días; en cambio, la solución de escoria experimenta el mismo efecto, sólo que de manera más lenta, alcanzándose

TABLa I.- Composición química de escorias Conox, \%

TABLE I.-Chemical composition of Conox slags, \%

\begin{tabular}{|l|c|c|c|c|c|c|c|c|}
\hline \multicolumn{1}{|c|}{$\mathrm{M}$} & $\mathrm{Si}_{2}$ & $\mathrm{Al}_{2} \mathrm{O}_{3}$ & $\mathrm{Ca} 0$ & $\mathrm{Mg} 0$ & $\mathrm{Mn} 0$ & $\mathrm{Fe} 0$ & $\mathrm{P}$ & $\mathrm{S}$ \\
\hline Horno & 11,17 & 0,87 & 42,96 & 5,88 & 6,86 & 28,75 & 0,58 & 0,05 \\
Patios & 12,42 & 1,21 & 44,08 & 5,89 & 6,05 & 26,0 & 0,52 & 0,14 \\
Historia & 13,71 & 0,93 & 45,49 & 6,74 & 6,29 & 23,29 & 0,63 & 0,05 \\
Ref. & 17,0 & 3,0 & 37,0 & 7,0 & 5,50 & 15,8 & 0,92 & 0,16 \\
\hline
\end{tabular}


TABLA II.-Densidad y superficie específica de los materiales utilizados

TABLE II.- Physical properties (Density and specific area) of used materials

\begin{tabular}{|l|l|c|}
\hline \multicolumn{1}{|c|}{ Material } & $\begin{array}{c}\text { Densidad, } \\
\mathrm{g} / \mathrm{cm}^{3}\end{array}$ & $\begin{array}{c}\text { Sup. esp, } \\
\mathrm{m}^{2} / \mathrm{g}\end{array}$ \\
\hline Suelo & 2,0965 & 2,27 \\
Cal agrícola & 2,2961 & 4,55 \\
Cal hidratada & 2,0126 & 9,26 \\
Carbonato de calcio & 2,5816 & 0,87 \\
Escoria: (-3,35+1,70) & 3,3662 & 0,576 \\
$(-0,60+0,425)$ & 3,0796 & 0,601 \\
$(-0,075+0,053)$ & 3,500 & 0,672 \\
$(-0,053)(-270$ mallas) & 3,5355 & 1,471 \\
\hline
\end{tabular}

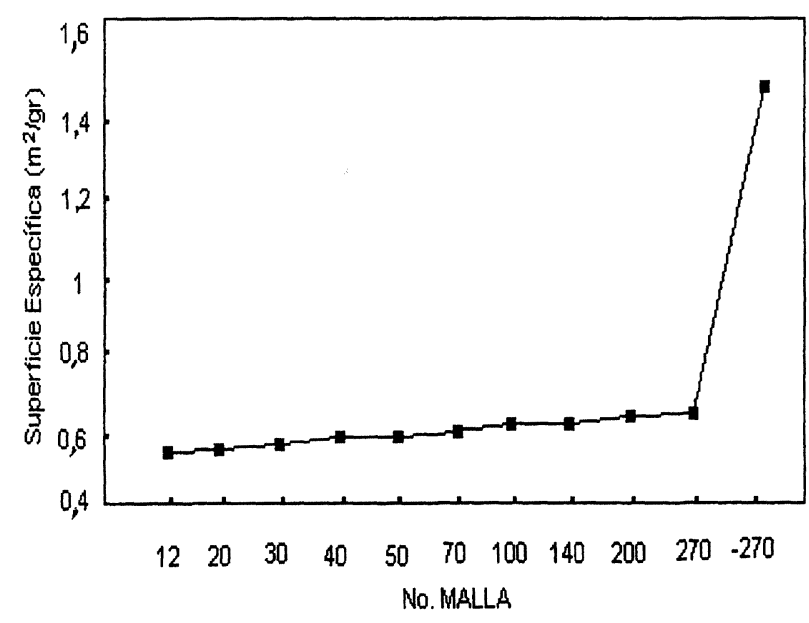

Fig. 2.- Cambio de la superficie específica con el nivel granulométrico.

FIG. 2.- Change of the specific area with particles size of materials.

el pH de 12 hasta los 16 días, con una estabilización posterior. Además, se comparó el cambio de composición de esta solución de escoria con el líquido sobrenadante en ella, obteniéndose un incremento de calcio de 14 a 528 ppm, y de magnesio de 6-19,2 $\mathrm{ppm}$. De lo anterior se evidencia que si bien es cierto que la capacidad de disolución de la escoria es muy baja, sí presenta condiciones alcalinizantes apreciables.

Continuando con dicho experimento, se analizó también el comportamiento del pH con relación al tiempo, para el tipo de modificador utilizado en suelos de cultivo ácido con adiciones de $0,2 \mathrm{~g} / 50 \mathrm{~g}$ de suelo, lo cual es equivalente a $8 \mathrm{t} / \mathrm{ha}$, según se reporta en la figura 3 .

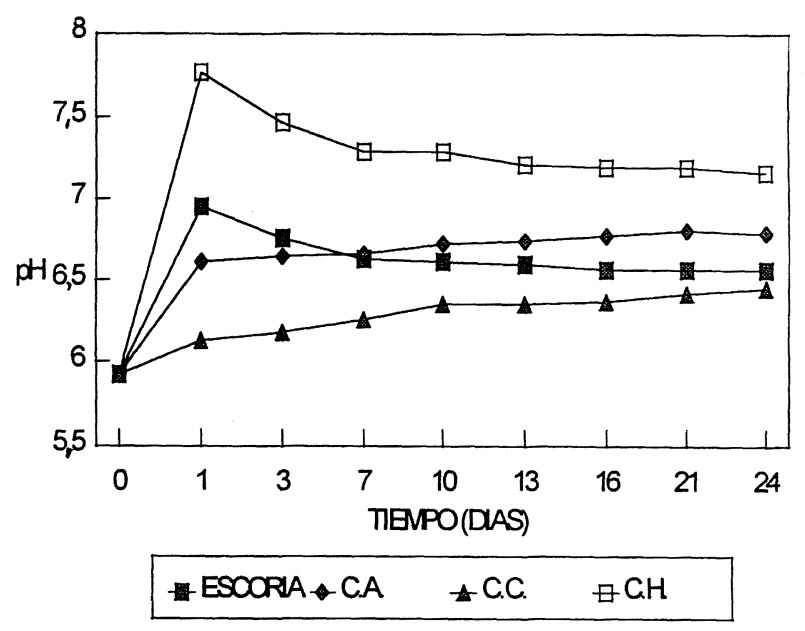

FIG. 3.- Cambio del pH en el suelo con los modificadores experimentados.

(C.A. = Cal agrícola; C.C. = Carbonato de calcio; C.H. = Cal hidratada; Escoria Conox)

FIG. 3.- Influence of time on the change of the $\mathrm{pH}$ in soils with conditioners.

(C.A. = Agricultural line; C.C. = Calcium carbonate; C.H. = Calcium hydrate; Conox slag)

De lo anterior se revela que la escoria se comporta de manera intermedia en cuanto a su $\mathrm{pH}$, comparada con el resto de los materiales básicos convencionales.

Una vez caracterizado el sistema, se procedió a evaluar la capacidad de aporte en micronutrientes de cada estabilizador en el suelo estudiado conforme a la tabla III .

De los resultados anteriores, se evidencia que la escoria presenta, en el intercambio iónico, una aportación en cationes calcio al suelo del orden del $56,6 \%$, comparado con el $71 \%$ que proporcionan en promedio los alcalinizantes. El catión magnesio se asimila en relación al contenido original en un

TABLA III.- Aporte de especies iónicas al suelo con las adiciones ensayadas

TABLE III.- Ionic species contribution to agricultural soil for the tested materials

\begin{tabular}{|c|c|c|c|c|c|}
\hline $\begin{array}{c}\text { Espec. } \\
\text { ppm }\end{array}$ & $\begin{array}{c}\text { Suelo } \\
\text { virgen }\end{array}$ & $\begin{array}{c}\mathrm{Cal} \\
\text { agrícola }\end{array}$ & $\begin{array}{c}\mathrm{Cal} \\
\text { hidratada }\end{array}$ & $\mathrm{CaC}_{3}$ & $\begin{array}{c}\text { Escoria } \\
\text { Conox }\end{array}$ \\
\hline $\mathrm{Ca}$ & 300 & 510 & 580 & 450 & 470 \\
$\mathrm{Mg}$ & 120 & 120 & 132 & 126 & 138 \\
$\mathrm{~K}$ & 16,8189 & 14,0157 & 14,0157 & 14,0157 & 14,0157 \\
$\mathrm{P}$ & 0,5481 & 0,8654 & 0,8366 & 0,7789 & 1,0963 \\
$\mathrm{Fe}$ & 0,7292 & 0,5925 & 0,6380 & 0,6836 & 1,1394 \\
$\mathrm{Mn}$ & 4,4285 & 6,4952 & 6,2000 & 7,6761 & 11,5143 \\
\hline
\end{tabular}


$15 \%$, contra el $5 \%$ en promedio de los estabilizadores comerciales.

El fósforo se incorpora en el suelo en un 50,87 $\%$, con los estabilizadores convencionales, y con la escoria se duplica su aporte al contenido inicial. El hierro se encuentra deficitario en un $87,49 \%$ con la influencia de los materiales convencionales, y en un $56,25 \%$ adicional al inicial, por efecto del uso de escoria. El manganeso, con la adición promedio de alcalinizantes, se asimila en el suelo en una proporción del 53,2 \%, comparado con el $160 \%$ que presenta la escoria. Lo dicho evidencia que, con el empleo de escorias se logra, además de estabilizar el carácter químico del suelo, conseguir incrementar el nivel de micronutrientes necesarios para el crecimiento del vegetal en lo general.

La velocidad de aporte de calcio y magnesio al suelo transcurre de manera más lenta cuando se utiliza escoria que con el resto de los materiales. Ello es debido a que las reacciones de disolución e hidrólisis de algunos de los componentes de la escoria son cinéticamente más lentas y termodinámicamente desfavorables (Fig. 4 y Tabla IV).

Por lo anterior, el proceso desde el punto de vista termodinámico es menos espontáneo, debido al consecuente carácter exotérmico débil de las reacciones, a excepción de la ferrita de calcio. La activación de este proceso, como de hecho sucede, es la influencia que presenta la intervención del correspondiente fertilizante que para este caso no fue valorado.

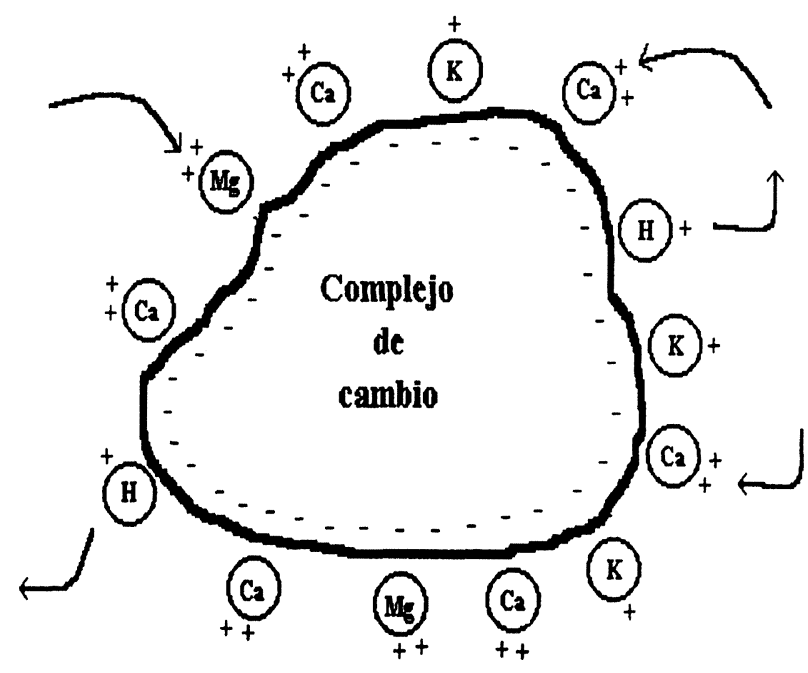

FIG. 4.- Esquematización de la formación de la bicapa eléctrica durante la hidrolización en la interfase escoria-suelo.

FIG. 4.- Scheme of the bi-electrical film formation during hydrolization reactions of the basic slags components.
TABLA IV- Comportamiento termodinámico del sistema escoria-suelo durante la hidrolización

TABLE IV.- Thermodynamical values of the hydrolyzation reactions of the basic slag components

\begin{tabular}{|c|c|c|}
\hline Reacciones & $\begin{array}{c}\Delta \mathrm{H}, \\
\mathrm{kcal} / \mathrm{mol}\end{array}$ & $\begin{array}{c}\Delta \mathrm{G}, \\
\mathrm{kcal} / \mathrm{mol}\end{array}$ \\
\hline$\beta \mathrm{Ca}_{2} \mathrm{Si}_{4}+4 \mathrm{H}^{+} \rightarrow$ & $-62,33$ & $-58,624$ \\
$\mathrm{SiO}_{2}+2 \mathrm{Ca}^{++}+2 \mathrm{H}_{2} \mathrm{O}$ & & \\
$\gamma \mathrm{Ca}_{2} \mathrm{Si}_{4}+4 \mathrm{H}^{+} \rightarrow$ & $-59,83$ & $-56,624$ \\
$\mathrm{Si}_{2}+2 \mathrm{Ca}^{++}+2 \mathrm{H}_{2} \mathrm{O}$ & & \\
$2 \mathrm{Ca} 0 \cdot \mathrm{Fe}_{2} \mathrm{O}_{3}+10 \mathrm{H}^{+} \rightarrow$ & $-112,955$ & $-71,795$ \\
$2 \mathrm{Ca}^{++}+2 \mathrm{Fe}^{+++}+5 \mathrm{H}_{2} \mathrm{O}$ & & \\
$\mathrm{CaOMg0SiO} \mathrm{M}_{2}+4 \mathrm{H}^{+} \rightarrow$ & & \\
$\mathrm{Ca}^{++} \mathrm{Mg}^{++}+\mathrm{Si} 0_{2}+2 \mathrm{H}_{2} \mathrm{O}$ & $-54,78$ & - \\
$\mathrm{Ca} 0+\mathrm{H}_{2} \mathrm{O} \rightarrow \mathrm{Ca}(0 \mathrm{H})_{2}$ & $-15,585$ & $-13,813$ \\
\hline
\end{tabular}

\section{CONCLUSIONES}

- El efecto de la mayor densidad en las escorias provoca un retraso en el proceso de incorporación de las especies iónicas al suelo de cultivo.

- El tamaño granulométrico en las escorias debe de considerarse, puesto que, tanto los materiales alcalinizantes y el propio suelo, denotan valores considerables en esta variable.

- La determinación del pH en soluciones acuosas de escoria y modificador demostraron el efecto alcalinizante de ésta, aunque su capacidad de disolución es reducida.

- La modificación del carácter químico del suelo $(\mathrm{pH})$ se logró competitivamente y de manera intermedia con las escorias de proceso, sin embargo éstas manifestaron una mayor aportación de elementos micronutrientes en comparación con los modificadores comerciales.

- El proceso de asimilación, durante el intercambio iónico en la interfase, se sucede con un carácter de exotermicidad muy débil y de poca espontaneidad, debido a las limitaciones de tipo cinético durante la hidrolización en el sistema escoria-suelo.

\section{Agradecimientos}

El autor expresa su amplio reconocimiento a los colaboradores en la realización de este trabajo. A los ingenieros Carlos Molina y Juan José González 
de la Empresa Sicartsa. Igualmente a los Ingenieros María Partida, José Pérez, Angélica Delgado y Víctor Talavera del Lab. de Química Agrícola del ITA No7.

Al M. en C. Sandra Bribiesca del I.I.M. de UMSNH. Finalmente, al Dr. Melchor Díaz Acevedo de PROPAM por el soporte financiero. A todos ellos nuestro agradecimiento por sus aportaciones técnicas y apoyo en la fase experimental de este estudio.

\section{REFERENCIAS}

(1) Willson, J. y Ladd, J. Rev. Acero, (2), 1994: 14-15.

(2) Rev. Siderurgia,(42), 1996: 31.

(3) Casares, F. y Guerra, E. Curso taller: La implementación de la Norma ISO-14001 en la empresa, ITESM,1996.

(4) Aldasoro, J. Extracción y procesamiento de escorias. Memoria Somisa. Argentina, 1987: 33-37.

(5) Formoso, A., Piret, J. y Balcazar, N. Slag utilization for recycling purpose. Palais Des Congres on Industrial Technologies. $5^{\circ}$ Conf. Memorie, (Bélgica), 1994: 3-16.

(6) Lopez, F., Clérigo, Z., Medina, F. y Formoso, A. Rev. Siderurgia, (49), 1989: 94-100.

(7) Whitten, K. Química General, $3^{\text {a }}$ rev. Mc Graw Hill, Nueva York, 1992: 256-259, 270-274.

(8) Herasymenko, P. Trans. Faraday.Soc., 34, 1938: 1.245.

(9) Temkin, M. Act. Phys. Chem., 20, URSS, 1945: 411.

(10) Fortland, T.J. Phys. Chem., 59, 1958: 152.

(11) Flood, H., Fortland, T. y GrJotheim, K. The physical Chemistry of metals, IMM, Londres, V1, 1953: 46.

(12) Flory, H.J. Chem. Phys., 10, 1942: 51-61.

(13) Frohberg, G, Kapoor y M. Germany, 41, 1970: 209-212.

(14) Blum, L. Stahl Eisen, 19 1901: 1024-1029.

(15) Platz, B. Stahl Eisen, 12, 1892: 2-8.

(16) Flood H. y Fortland, T.J. Act, Chem. Scand., 1, 1947: 592, 781, 790.

(17) Whiteley, J. Proc. Cleveland Inst. Eng., 59, 1922-1923.

(18) Turkdogan, E. Phys. Chem. Steelmaking, Thermochem. Thermodyn. Vol. II, 1970: 15-20.

(19) Winkler, T y Chipman, J. Trans. AIME, 167, 1946: 111.

(20) Darken, L. y Larsen, B., Trans. AIME, 150, 1942: 87.

(21) Herty, C., Christopher, C., Freemann, H. y SANDERSEn, J. Min. Met. Inv. Bull. (68), 1934:

(22) Grant, N. y Chipman, J. Trans. AIME, 167, 1946: 134.

(23) Hatch, G. y Chipman, J. Trans. AIME, 185, 1949: 274.
(24) Chipman, J. y Hatch, G. Trans. AIME, 167, 1946: 134.

(25) Bruckner, R. y Glastech, B. 37, 1964: 413.

(26) JindRICH, P. Catálogo "Ocelarske strusky". Ostrava (Checoslovaquia) 1980: 1-5:

(27) Toop, G. y SAmis, C. Trans. TMS-IME, 224, 1962: 878.

(28) Bockris, J. y RedDY, A. Modern ElectroChemestry. Nueva York, Vol. 1, 1970: 23-29.

(29) Romero, J. Termodinámica de la desulfuración de aceros. Curso, I.PN, México, 1994: 12.

(30) Bohn, H., McNeal, B.O. y Connor, G. Química del suelo. Limusa, Vol 1, 1993: 123-153.

(31) Thompson, L. El suelo y su fertilidad. Ed. Reverté España, 1974: 14-12.

(32) Aguilera, M. y Martinez, R. Relaciones Planta-SueloAtmósfera. Chapingo. México, 1990: 63-97.

(33) Pritchett, W. Suelos forestales, propiedades, conservación y mejoramiento. Limusa. México, 1986: 140.147.

(34) Geiseler, J. Stahl Eisen, 111 (1), 1991: 133-138-43.

(35) NAGAI, K. y SASAKI, M. Trans. ISIJ, 25 (7), 1985: 621625.

(36) ОнI, H. Bull. ISIJ. 22 (8) 1983: 695-701.

(37) BlucK, G. y GeISELER, J. Stahl Eisen, 100 (3), 1980: 118 123.

(38) GinSKIO, Ya. F. Comprehensive processing of ironmaking and steelmaking slags. Met. N111, 1978: 38-40.

(39) Matyas, A. Utilization Steelmaking Slags ISE, 55 (8), 1978: 29-30.

(40) Li, H., SuITO, H. y TokudA, M. Trans. ISIJ, 24 (4), 1984: 1079-1087.

(41) José, F. Escorias Siderúrgicas. Inst. Argentino de Siderurgia. Buenos Aires. (3), 1988: 3.

(42) GutT, W. Chem. Ind., 1972: 439-447.

(43) InOUE, Suito, H. ISIJ Int., 35 (3), 1995: 272-279.

(44) Crawford, C. y Burn, K. ASCE, 1969: 1325-1334.

(45) Formoso, A., López, F., Medina, F., Balcázar, N., Ruiz y LABuru, J. Utilización agrícola de escorias de convertidor al oxígeno. XV Simposio Nac. Siderurgia. México, 1993: 5.1-5.12

(46) López, F., Clérigo, Z., Medina, F. y Balcázar, N. Experiencia española en la utilización de escorias. $7^{\circ}$ Congreso Nac. de Ciencia y Tecnología. Madrid, Vol.1, 1990: 171-180.

(47) Waelkens, G. y Carmine C. del, Generación y propiedades de las escorias siderúrgicas. Memoria. SOMISA. Argentina, 1987: 16.

(48) Cartas edafológicas INEGI. México, 1981: E.13-3, E 136, E 14-4; F.13D (78, 79, 86-89); F 14C (71, 72, 81-86).

(49) Guías de carta interpretativas cartográficas. INEGI. México. 1981. 